\title{
Influence of a commercial antithrombotic filter on the caval blood flow during neutra and Valsalva manoeuvre
}

\author{
M. Nicolas \\ Aragón Institute of Engineering Research (I3A). \\ Universidad de Zaragoza. \\ C/María de Luna s/n, E-50018 Zaragoza, Spain \\ B. Lucea \\ Aragón Institute of Engineering Research (I3A). \\ Universidad de Zaragoza. \\ C/María de Luna s/n, E-50018 Zaragoza, Spain
}

\begin{abstract}
A. Laborda
Grupo de Investigación Técnicas de Mínima Invasión (GITMI), Faculty of Veterinary, Universidad de Zaragoza, C/Miguel Servet 177, E-50013 Zaragoza, Spain
\end{abstract}

\section{E. Peña}

Aragón Institute of Engineering Research (I3A).

Universidad de Zaragoza.

C/María de Luna s/n, E-50018 Zaragoza, Spain

Centro de Investigación Biomédica en Red en Bioingeniería Biomateriales y Nanomedicina (CIBER-BBN)

\author{
M. A. De Gregorio \\ Grupo de Investigación Técnicas de Mínima Invasión (GITMI), \\ Faculty of Veterinary, Universidad de Zaragoza, \\ C/Miguel Servet 177, E-50013 Zaragoza, Spain
}

\section{A. Martínez}

Aragón Institute of Engineering Research (I3A).

Universidad de Zaragoza.

C/María de Luna s/n, E-50018 Zaragoza, Spain

Centro de Investigación Biomédica en Red en Bioingeniería Biomateriales y Nanomedicina (CIBER-BBN) 
Department of Mechanical Engineering, Energetics and Materials, Public University of Navarra, Campus Arrosadía, E-36001, Pamplona. Aragón Institute of Engineering Research (I3A). Universidad de Zaragoza.

C/María de Luna s/n, E-50018 Zaragoza, Spain

Centro de Investigación Biomédica en Red en Bioingeniería Biomateriales y Nanomedicina (CIBER-BBN)

Email: mauro.malveeunavarra.es

\begin{abstract}
Anticoagulants are the treatment of choice for pulmonary embolism. When these fail or are contraindicated, vena cava filters are effective devices for preventing clots from the legs from migrating to the lung. Many uncertainties exist when a filter is inserted, especially during physiological activity such as normal breathing and the Valsalva manoeuvre. These activities are often connected with filter migration and vena cava damage due to the various related vein geometrical configurations. In this work we analysed the response of the vena cava during normal breathing and Valsalva manoeuvre, for a healthy vena cava and after insertion of a commercial GüntherTulip ${ }^{\circledR}$ filter. Validated computational fluid dynamics $(C F D)$ and patient specific data are used for analysing blood flow inside the vena cava during these manoeuvres. While during normal breathing the vena cava flow can be considered almost stationary with a very low pressure gradient, during Valsalva the extravascular pressure compresses the vena cava resulting in a drastic reduction of the vein section, a global flow decrease through the cava but increasing the velocity magnitude. This change in section is altered by the presence of the filter which forces the section of the vena cava before the renal veins to keep open. The effect of the presence of the filter is investigated during these manoeuvres showing changes in wall shear stress and velocity patterns.
\end{abstract}

\title{
1 Introduction
}

Deep vena cava thrombosis is a potentially deathly disease consequent to a pulmonary thromboembolism (TEP). This is the third cause of death in hospitals with an incidence of 1 case/1000 inhabitants per year [1]. TEP consists of an obstruction of the pulmonar artery due to a blood clot traveling in the cardiovascular system. The most common available alternatives for treating TEP are anticoagulation therapy or inferior vena cava filters (IVCF). Insertion of an IVCF is recommended when anticoagulants are contraindicated or when a patient is unresponsive to anticoagulants [2,3]. Currently, filters are used particularly in cases of high risk of pulmonary embolism or when the pulmonary functional reserve is low. These include, among others, the Greenfield ${ }^{\circledR}$ filter (Boston Scientific Incorporated, Watertown, MA), Vena Tech ${ }^{\circledR}$ filter (B. Braun/VenaTech, Vanston IL), Simon-Nitinol ${ }^{\circledR}$ filter (Bard Peripheral Technologies, Murray Hill, NJ), TrapEase ${ }^{\circledR}$ filter Cordis (Miami Lakes, FL), Günther-Tulip ${ }^{\circledR}$ filter (Cook Medical Incorporated, Indianapolis, IN) and Recovery-Nitinol ${ }^{\circledR}$ filter (Bard Peripheral Technologies, Murray Hill, NJ). Ideally, an intravascular filter should allow the normal venous blood flow while being able to capture the blood clot without causing lysis [4]. Inferior vena cava filters should be easy to insert and extract, provide a sufficient radial force to avoid migrations, possess high elasticity and elastic recoverability without material fatigue, be well tolerated without damaging vein walls and have an optimal biocompatibility in order to avoid foreign-body reaction, granulation tissue and/or or precipitate infection [5-8]. As documented in the literature, there is to date no filter that ideally meets all these requirements. One very important aspect is how the blood flow changes in the presence of an IVC filter before and after clot capture $[9,10]$. Even though some studies have analysed how the hemodynamic is affected by the insertion of a filter and additionally by the presence of a blood clot [11-15], this has usually been performed only for simple cava geometries and only during normal conditions, adding lumbar curvature [16] or comparing rest and exercise conditions [17]. [18] performed a patient-specific CFD analysis studying the influence of filter location with and without embolus and comparing normal and exercise conditions. In these studies, the influence of the filter position was studied and the hemodynamics changes due to the possible capture of a blood clot were detailed. On the other hand, other authors have also experimentally attempted to define the clot capturing efficiency of commercial filters in vitro [19]. Unfortunately, although effective for studying the vena cava and filter flow dynamics, these in vitro experiments have their limitations. Physical models in vivo and in vitro do not always allow the study of certain scenarios due to limitations of the experimental setup [4]. The effect of the filter during the Valsalva manoeuvre on the other hand has not yet been quantified. While normal breathing corresponds to neutral vena cava conditions, the Valsalva manoeuvre consists of a forced exhalation against a closed glottis. Commonly, this manoeuvre can be undertaken during forced defecation or forceful coughing as well as by lifting a 

pressure occurs while cardiac frequency reduces, venous pressure increases and vena cava flow diminishes [20,21]. A prolonged Valsalva manoeuvre, especially in adults may result in several complications. These may include, among others, unphysiological pressure increase in cerebral arteries which may lead to arterial local ruptures in patients with aneurysm, or pneumothorax and cardiac infarction [22]. As a consequence of a Valsalva manoeuvre, the vena cava hemodynamics also radically changes. In particular, the increase in intrathoracic pressure causes a compression of the inferior and superior vena cava, obstructing the venous return and causing a decrease in the arterial pressure. The Valsalva manoeuvre, while physiological, is also frequently adopted in hospitals to clarify patient symptoms as well as to examine the Eustachian tube function and/or to manage paroxysmal supraventricular tachycardia. Other uses for the manoeuvre in clinical practice are related to venous and intracardiac pressure changes. These include the evaluation of the left ventricular function and the diagnosis of congestive cardiac failure [23], the facilitation of the insertion of central venous catheters, the enhancement of lower limb color Doppler flow imaging and the ultrasonographic detection of biliary obstruction [22].

Computational fluid dynamics (CFD) can be considered a powerful tool for studying blood flow in the vena cava, starting from medical images in the absence and presence of filters. Local hemodynamics with clot capturing can be used to improve clinical techniques, being easy, flexible and reproducible numerical models, which are complementary to current in vitro efforts $[4,24]$. These models are able to generate detailed and comprehensive information of the relevant flow variables, which are normally not easy to obtain in vitro or in vivo.

One of the principal disadvantages of a metallic filter is the high risk of fibrosis due to the cellular proliferation around the filter struts [25]. This aspect complicates the filter extraction, while at the same time it may avoid filter migration. For this reason it is clear that further investigations are needed in order to understand how the insertion of a filter affects the vena cava and in order to establish which aspect may play the most important role. This investigation should consider especially the Valsalva manoeuvre which is associated to drastic geometrical changes such as collapse of the vein [26] and may conduct to abrupt penetration of the filter leg through the vena wall [25]. The consequences of the filter presence during this manoeuvre is especially challenging. For the aforementioned reasons the analysis of a filter is a significant and necessary issue in the clinical treatment of TEP, considering the uncertainties affecting the relationship between the vena cava and the device. In this work we use CFD technique to analyse two vena cava physiological conditions, i.e. neutral and Valsalva conditions, as well as the effect of the insertion of the commercial filter Günther-Tulip ${ }^{\circledR}$ (Cook Medical Incorporated, Indianapolis, IN) for studying its impact on the overall vena cava hemodynamics. The numerical model is based on CT images of human patients during the aforementioned conditions. The novel aspect of the present study is twofold: first, it analyses how the presence of the ICV filter affect the geometry and hemodynamics of the vena cava flow in neutral and Valsava manoeuvre conditions. Previous numerical studies in the field are limited to neutral or exercise conditions only [18, 27, 28]. Second, the analysis of the presence of a filter improves knowledge in the field and is necessary for designing better adapted and personalized medical devices in order to improve clinical outcomes.

\section{Materials and Methods}

\subsection{Vena cava model: Neutra and Valsalva conditions}

Human vena cava geometry was firstly considered under neutral and Valsalva conditions. CT images from a patient before filter implantation (Patient A) were obtained in 64-slices with a Brilliance CT scanner (Philips Electronics. Amsterdam, The Netherlands) with 100-120 cc. of intravenous ioversol (Optiray Ultraject 240, Covidien, Barcelona, Spain) and reconstructed with a slice thickness of $5 \mathrm{~mm}$. In particular, low dose CT protocols (120 $\mathrm{kV}$ and $30 \mathrm{mAs}$ ) were used. The patient was instructed to perform the Valsalva under reproducible conditions. Three valid manoeuvres with low dispersion between the recorded values were considered and the mean parameters were used in the CFD simulations, both for neutral and Valsalva conditions. The Valsalva manoeuvre is divided in four main stages [21]. In phase I or early Valsalva the blood is expelled from the thoracic vessels by the increase in intrathoracic pressure. Phase II or late Valsalva consists on the increase in intrathoracic pressure that causes a reduction of venous return, lowering the cardiac output. In phase III or early recovery as intrathoracic pressure suddenly drops there is pooling of blood in the pulmonary vessels, causing a further drop in blood pressure. Finally in phase IV or late recovery with venous return restored there is an overshoot, as compensatory mechanisms start to operate. The vena cava geometry was recorded during the whole process although only phases I and II were considered, where the geometry remains unalterable. The DICOM files from the scan were processed using the commercial software MIMICS (Materialise Software, Leuven). These files provided a clear picture of the vessel lumen. With aim to generate a three-dimensional model, a non-automatic segmentation of the CT scans was accomplished to determine the real geometry of the vena cava. In each scan, the vessel lumen was manually filled till reaching the vena cava wall. At this stage, the difference in grey tones between lumen and vessel allows the software to fit the caval section according with the image pixel and helping the manual filling. In particular, the software MIMICS converted the medical images unities (Hounsfield unities) to its own grey tones unities when the images are firstly imported in the software. The obtained grey tones value range at this stage was $-1024 \div 3071$ unities of MIMICS. From this range, for the manual segmentation, we have restricted the thresholds to $1814 \div 2789$ unities that helps selecting the vena cava lumen. After that, the segmentation was performed 
(a)
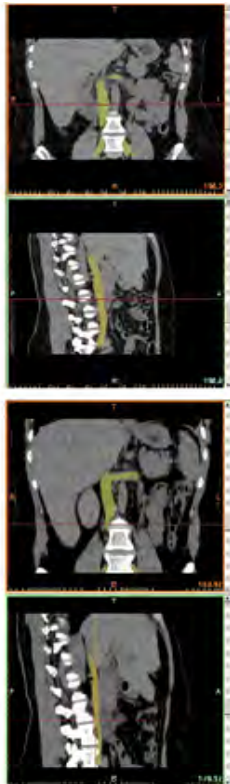
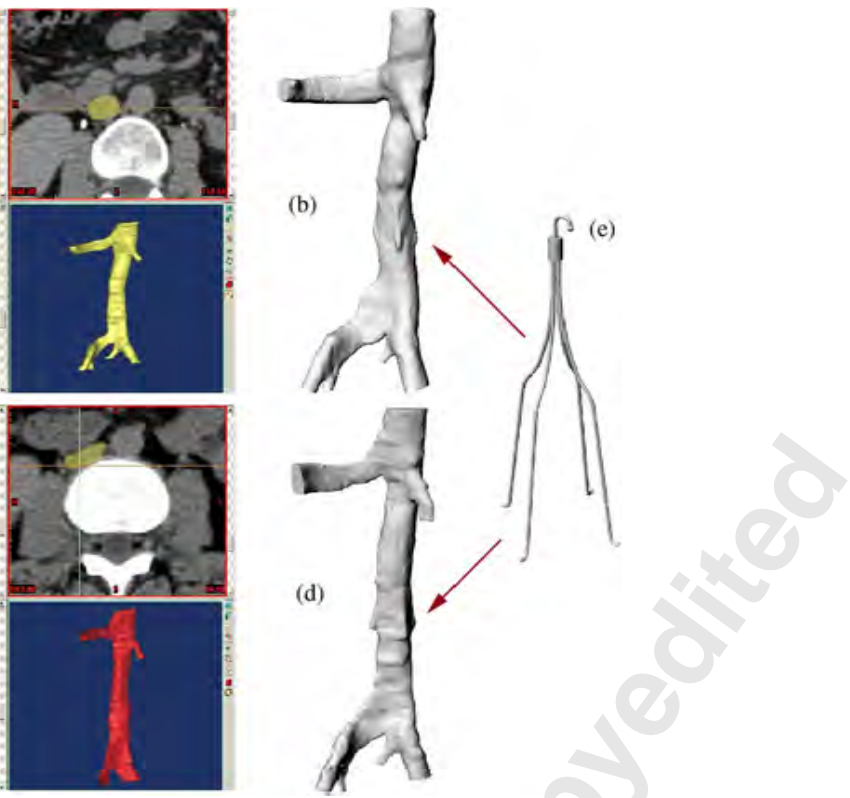

Fig. 1. CAD model of vena cava during normal breathing (a), normal breathing with filter (b), Valsalva without filter (c) and Valsalva with filter (d). Subfigure (e) shows the Günther-Tulip ${ }^{\circledR}$ filter CAD model.

manually, section by section considering each CT scan. From this segmentation a stereolithography (STL) file was finally obtained. For improving the noisy geometry that presented non physiological peaks, an additional smoothing was automatically performed allowing the interpolation of the nodal net points of the STL along the computed surfaces. A smoothed STL file of the segmented geometry yet containing the geometrical three-dimansional model was finally exported. These data were imported in the commercial software Rhinoceros (Robert McNeel \& Associates). In this way, the three-dimensional model of the vena cava for normal breathing (as well as for Valsalva condition) was prepared for the mesh generation. In this software, the inlet and outlets surfaces were rebuilt and treated as separated surfaces for imposing later the boundary conditions. Finally, using Ansys Icem (Ansys Inc. Software, Canonsburg, PA, USA) we obtained the numerical grid composed of $3 * 10^{6}$ tetrahedral elements. In order to guarantee that the numerical results were grid independent a mesh independence study was carried out prior to the simulations. Velocity and wall shear stress profiles at different vena cava locations were compared for different grid sizes. This comparison clearly demonstrated that meshes finer than $3 * 10^{6}$ elements increased the computational time without adding precision to the solution.

\subsection{Filter model: Neutra and Valsalva conditions}

As described in the previous sections, the presence of the commercial filter Günther-Tulip ${ }^{\circledR}$ (Cook Medical Incorporated, Indianapolis, IN) inside a vena cava was studied during neutral and Valsalva conditions. This filter presents four legs to hold the filter in-place and a hook attached to the top of the filter for allowing retrieval of the device by means of a sheath inserted into the jugular vein. Fastening barbs at the end of the four straight legs ( see Figure 1 - (e)) provide resistance to migration in the IVC. Moreover, this filter has a length of $40 \mathrm{~mm}$ and a leg thickness od $0.1 \mathrm{~mm}$. CT images of a new patient after filter implantation (Patient B, different from that of the reconstruction without device) were obtained using the same procedure described in the previous subsection. The medical images were obtained with the same precision during neutra and Valsalva providing the location of the filter which was separately reconstructed and inserted after the geometrical model reconstruction at the exact position provided by the images. In particular, following the dimensions of the filter, this was adapted to the vena cava using the intersections of the struts with the vena cava wall which was clearly recognized through the images. Figure 1 depicts the vena cava and the filter in the expanded configuration during neutral condition without (a) and in the presence of the filter (b), and, during the Valsalva manoeuvre without (c) and in the presence of the filter (d), respectively. The CAD model of the Günther-Tulip ${ }^{\circledR}$ filter is shown in Figure 1 - (e). In Figure 2 the section of the vena cava is shown at the filter location for both conditions of neutral and Valsalva. For the purpose of comparison, this cross-section is also shown in the absence of a filter during the aforementioned manoeuvres. It can be clearly seen that while in neutral condition the section is almost circular, during Valsalva the extravascular pressure compresses the vena cava making the section elliptical (see Figure 2 upper panels). The massive influence of the filter during these manoeuvres is also visible. The filter imposes a square section in the region of its deployment during neutral and Valsalva conditions completely preventing the collapse of the vena cava (see Figure 2, lower panels). 

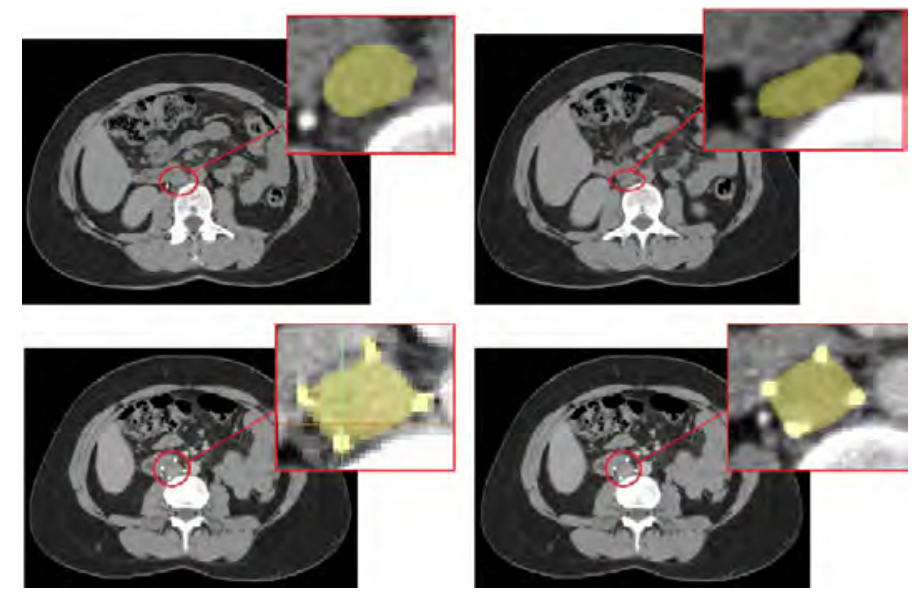

Fig. 2. Vena cava sections in the absence and in presence of the Günther Tulip ${ }^{\circledR}$ filter during normal breathing (left subfigures) and Valsalva (right subfigures).

Using Ansys Icem (Ansys Inc. Software, Canonsburg, PA, USA) we obtained the numerical grid of the vena cava models after deployment of the device. Due to the presence of the filter, the computational meshes increased their sizes in order to discretize the regions around the filter struts with the degree of precision required. The final grids were composed of over $5 * 10^{6}$ tetrahedral elements. In Figure 3 the details of the models after filter deployment are sketched and the regions around the filter are depicted. The filter mesh is also shown with a close-up view of its struts. As described before, also in this case, in order to guarantee that the numerical results were grid independent a mesh independence study was carried out. As previously explained, velocity and WSS profiles at different vena cava locations and on a line along the vena cava surface respectively were compared for different grid sizes. This comparison clearly demonstrated that meshes finer than $5 * 10^{6}$ elements increased the computational time without adding precision to the solution.

\subsection{Grid independence study}

In order to establish grid independence for the numerical results presented in this work, we conducted additional computations with different grid sizes. In particular, we considered the geometry of the vena cava during normal breathing with and without filter. These models were considered reasonably representative also for the manoeuvre of Valsalva. For normal breathing with filter, we tested three more grid sizes of about $1 * 10^{6}, 2.5 * 10^{6}$ and $1 * 10^{7}$ (in addition to that of $5 * 10^{6}$ ) at the same flow rate respectively (see next section). Velocity and WSS profiles at different vena cava locations and on a line along the vena cava surface respectively depicted in the Figure 4 and 7 were compared for these grid sizes. The relative error between these grids in term of peak velocity profile showed to be less than $3 \%$ between grids of $5 * 10^{6}$ and $1 * 10^{7}$. Plots of the WSS along a line depicted on the vena cava surface for the used four meshes with increasing density showed no substantial quantitative difference among results while higher with respect to the case of the velocity evaluation. Wall shear stress data from selected sections showed maximal variations of about 5\%. This results is similar to the findings of Stewart and coworkers [13], even they used a different filter type and they included the clot in their study. From these results, we can conclude that the chosen mesh density is fine enough to capture the desired details of the caval flow. Taking into account
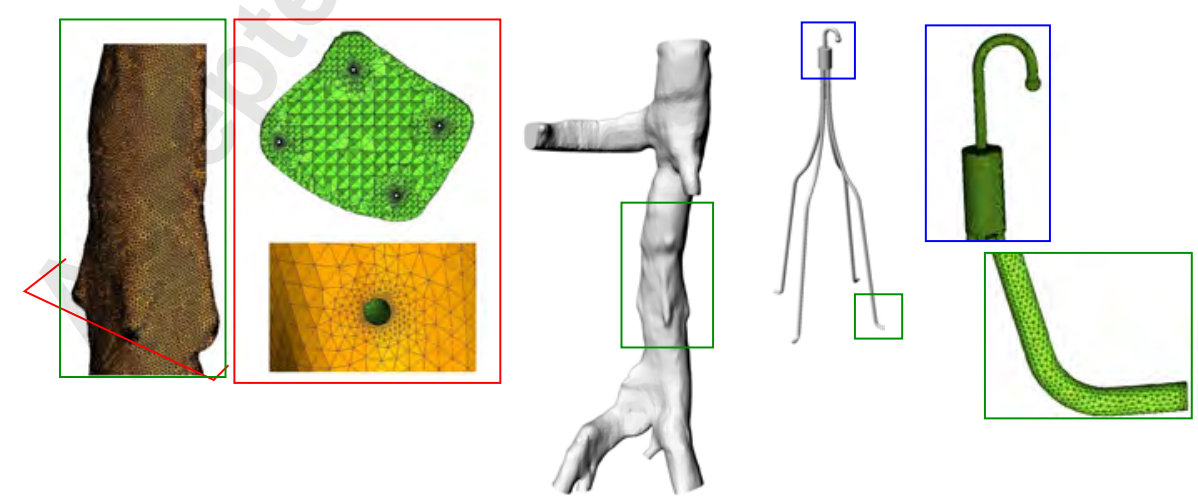

Fig. 3. Details of the vena cava grid after device deployment (as an example during normal breathing): close up view of the filter struts and the filter middle section. 


\begin{tabular}{|l|l|l|l|l|l|l|}
\hline & $\begin{array}{l}\text { Area }\left[\mathrm{mm}^{2}\right] \\
\text { Neutra }\end{array}$ & $\begin{array}{l}\text { Area }\left[\mathrm{mm}^{2}\right] \\
\text { Valsalva }\end{array}$ & $\begin{array}{l}\text { Flow }[L / \mathrm{min}] \\
\text { Neutra }\end{array}$ & $\begin{array}{l}\text { Flow }[L / \mathrm{min}] \\
\text { Valsalva }\end{array}$ & $\begin{array}{l}\text { Vel. }[\mathrm{m} / \mathrm{s}] \\
\text { Neutra }\end{array}$ & $\begin{array}{l}\text { Vel. }[\mathrm{m} / \mathrm{s}] \\
\text { Valsalva }\end{array}$ \\
\hline \hline Inlet 1 & 112.2 & 37.31 & 0.38 & 0.27 & 0.056 & 0.135 \\
\hline Inlet 2 & 44.38 & 26.78 & 0.15 & 0.105 & 0.055 & 0.066 \\
\hline Inlet 3 & 15.18 & 13.02 & 0.122 & 0.087 & 0.134 & 0.11 \\
\hline Inlet 4 & 185.15 & 61.03 & 0.925 & 0.655 & 0.084 & 0.18 \\
\hline Inlet 5 & 146.12 & 79.60 & 0.5 & 0.355 & 0.057 & 0.074 \\
\hline Inlet 6 & 12.16 & 12.81 & 0.023 & 0.017 & 0.032 & 0.02 \\
\hline
\end{tabular}

the increase of computational cost between consecutive grids, within the scope of the present work, all of the presented results related to the vena cava in the presence of a filter were obtained from computations using $5 * 10^{6}$ meshes. A similar comparison was performed also for the vena cava during normal breathing in absence of the filter. In this case we tested grid sizes of about $1 * 10^{6}, 3 * 10^{6}, 6 * 10^{6}$ and $9 * 10^{6}$ and we obtained independence with the aforementioned criterion for the grid size of about $3 * 10^{6}$.

\subsection{Blood flow model and boundary conditions}

The vena cava blood flow can be considered as stationary, the pressure gradient and the related velocities being very low in comparison with arterial blood flow [4,14]. For the considered numerical simulations, blood was assumed to be Newtonian $\left(\rho=1060 \mathrm{~kg} / \mathrm{m}^{3}, \mu=0.003528 \mathrm{~Pa} \cdot \mathrm{s}\right)$ and incompressible under steady conditions [4,12]. In addition, the Reynolds number, $\operatorname{Re}=\rho U D / \mu$, based on the inlet diameter $D$ and mean velocity flow $U$ of the IVC is $R e \approx 500$ and $R e \approx 1200$ for neutral and Valsalva conditions respectively.

The fluid grids were imported in the commercial software package Ansys CFX (Ansys Inc. Software, Canonsburg, PA, USA) with which the fluid dynamics (CFD) computations were carried out. The numerical approach used by this software is extensively explained in the literature and the details are given in [29].

The simulations performed in this study were carried out using the 16 nodes, Dual Nehalem (64 bits), 16 processors cluster with a clock speed of $2.33 \mathrm{GHz}$ and $32 \mathrm{~Gb}$ memory for each node. The convergence criteria used in the simulations performed in this study was $1 * 10^{-8}$. The boundary conditions for neutra and Valsalva manoeuvres are sketched in the Figure 4 - (b). Intravascular blood pressure data were collected during neutra and Valsalva through venous catheter insertion. Systolic, diastolic and mean pressures were recorded at suprarenal (SR) position with a Vitara PA 8060 Pressure Monitor (Draeger Medical Hispania, Madrid, Spain). A $100 \mathrm{~cm}, 5 F$ pig-tail catheter (Cook Medical, Limerick, Ireland) was used, obtaining measurements at the aforementioned location. Blood pressure corresponding to mean value was imposed at the vena cava outlet while blood flow was imposed at iliac and renal veins. Velocity flow during neutra and Valsalva were measured in vivo at the suprarenal vena cava location. An ALOKA UltraSound system (Hitachi Medical Systems Europe) was used to

(a)

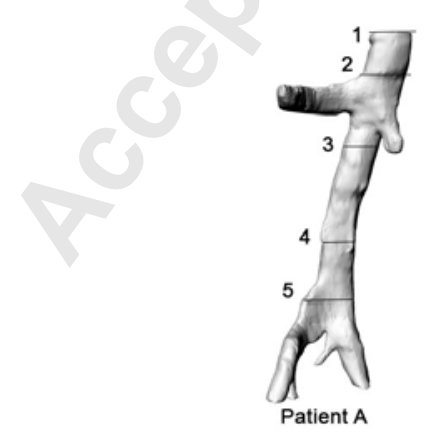

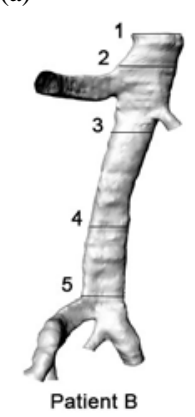

(b)

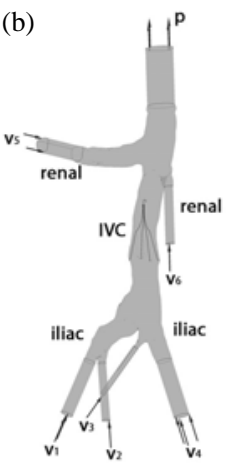

Fig. 4. Geometry for patient A and B showing the locations where the IVC areas have been studied (subfigure (a)). Boundary conditions for neutra and Valsalva manoeuvres (subfigure (b)): mixed measured pressure/velocity conditions are used. 
Copyright (measure hlow conditions during neutral and Valsalva manoeuvre. The mean flow during phases I and II of Valsalva was calculated. This flow was then adapted and divided in order to be applied at renal and iliac veins using the Murray law [30]. Table 1 and Figure 4 - (b) summarizes and shows respectively the applied inflow boundary conditions at the extremities of all CAD models. Table 2 shows the average pressure measured in different points of the vena cava in distinct Valsalva manoeuvres. Only the pressure in the suprarenal position was imposed as outlet boundary condition. The high pressure into the IVC reached during Valsalva manoeuvre is a direct consequence of the increase in intra-abdominal pressure. It has to be noted that velocity boundary conditions were applied at model extensions. These were added to the model in order to guarantee fully developed flow in the region of interest of the vena cava. Table 1 also shows the values of the areas for all the boundaries to remark very clearly how the area is reduced during Valsalva manoeuvres, creating a notable increase of the velocity in all the boundaries, while the flow rate is decreased by a factor of 0.71 as it has been previously seen on [20,21]. If the flowrates of all the inlets at neutra condition are added, a flowrate of $2.1 \mathrm{~L} / \mathrm{min}$ is obtained defining the suprarenal flow rate. This value is in accordance with other flow rate used by other authors during normal breathing $[11,16-18,21,28]$ indicating that the boundary conditions used in our model are coherent.

Table 2. Measured pressures at different locations during neutra and Valsalva manoeuvres (outflow conditions).

\begin{tabular}{|l|c|}
\hline & Pressure $[\mathrm{mmHg}]$ \\
\hline \hline Valsava Suprarenal & 14.21 \\
\hline Valsava Infrarenal & 40.68 \\
\hline Valsava Juxtarenal & 27.65 \\
\hline Neutra Suprarenal & 1 \\
\hline
\end{tabular}

\section{Results}

In order to fully understand the results obtained for the four models computed, it is important first to take a closer look at some vena cava area sections. As it was previously described, two different patient specific geometries were used for this work; one for the IVC during neutra and Valsalva conditions with no filter inserted, and a second one during neutra and Valsalva conditions with the filter inserted. Figure 4 - (a) shows the mentioned patient specific geometries along with the locations where the section areas have been analysed. Location 1 and 2 belong to the suprarenal region of the vena cava, while locations 3, 4 and 5 belong to the infrarenal region of the vena cava. Table 3 shows the areas in $\mathrm{mm}^{2}$ at all the locations for the four models computed. In particular, location 4 belongs to the section where the struts of the filter are located.

The areas for both neutra condition with and without filter at this location are $339.2 \mathrm{~mm}^{2}$ and $358.8 \mathrm{~mm}^{2}$ respectively. If we assume that this section is a perfect circumference their diameters would be $10.39 \mathrm{~mm}$ and $10.67 \mathrm{~mm}$ respectively, as it would be expected from the filter dimensions. On the other hand by looking at the area sections at location 4 for the Valsalva models, smaller areas and diameters are obtained as it can be seen on Table 3. This is due to the increase in intrathoracic pressure that is too high making the filter struts to compress a little bit. It is also important to point out that the geometry

Table 3. Area of the locations shown in Figure 4 - (a) for the four patient specific geometries and percentage change between natural breathing (considered as control value for comparison purpose) and Valsalva manoeuvre.

\begin{tabular}{|l|c|c|c|c|c|c|}
\hline \multirow{2}{*}{ Area $\left(\mathrm{mm}^{2}\right)$} & \multicolumn{5}{|c|}{ Patient A } & \multicolumn{4}{c|}{ Patient B } \\
& control - filter & Valsalva filter & \% change & control - no filter & Valsalva no filter & \% change \\
\hline \hline Area 1 & 388.95 & 106.35 & 73.43 & 512.10 & 204.28 & 60.11 \\
\hline Area 2 & 446.27 & 136.70 & 69.37 & 562.08 & 301.61 & 46.34 \\
\hline Area 3 & 342.20 & 79.60 & 76.74 & 373.55 & 216.06 & 42.16 \\
\hline Area 4 & 339.2 & 149.94 & 55.8 & 358.8 & 151.3 & 57.83 \\
\hline Area 5 & 390 & 92.85 & 76.19 & 251.36 & 137.02 & 45.49 \\
\hline
\end{tabular}




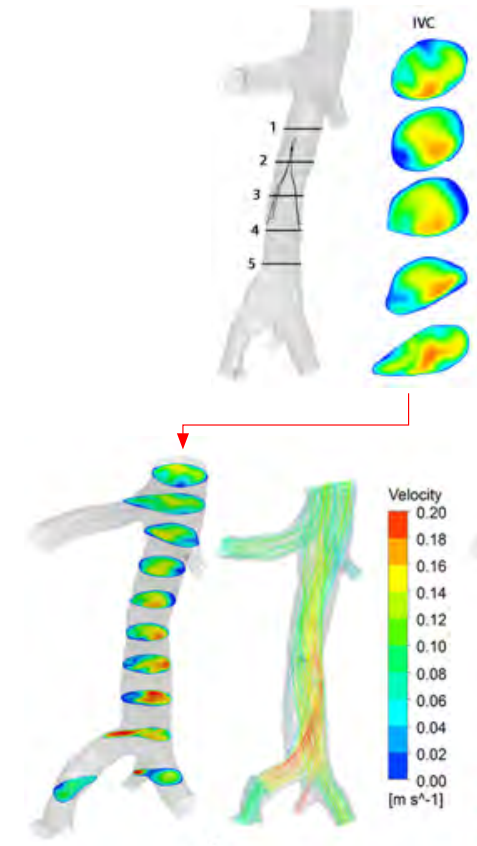

a
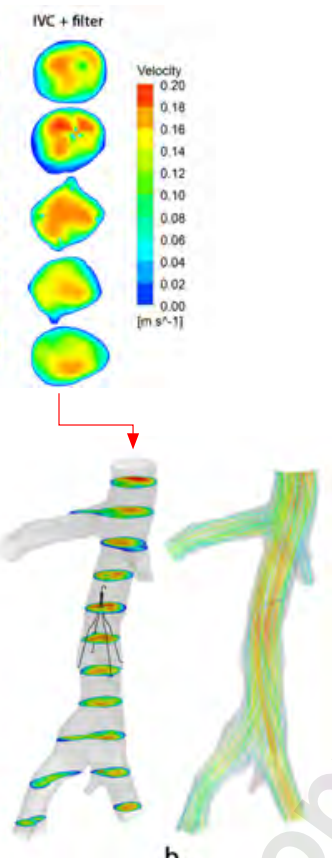

b

Fig. 5. Comparison between the vena cava fluid dynamics without filter (a) and after device deployment (b) in neutral conditions. On the upper panel, velocity magnitude is displayed on transversal cut planes, on the lower panel three dimensional streamlines are shown.

from patient B has a greater diameter than that of patient A (see Figure 4 - (a)). This can be also seen in Table 3 where it is shown how the areas for patient B are greater than for patient A in both neutra and Valsalva conditions. In both patients it is clearly seen how the area decreases during Valsalva compared to neutra. This information regarding the area along different sections of the IVC will be important when understanding the velocity results obtained. The maximal opening of the filter in neutra condition is $339.2 \mathrm{~mm}^{2}$ at the filter legs location. During Valsalva manoeuvre it is evident how this

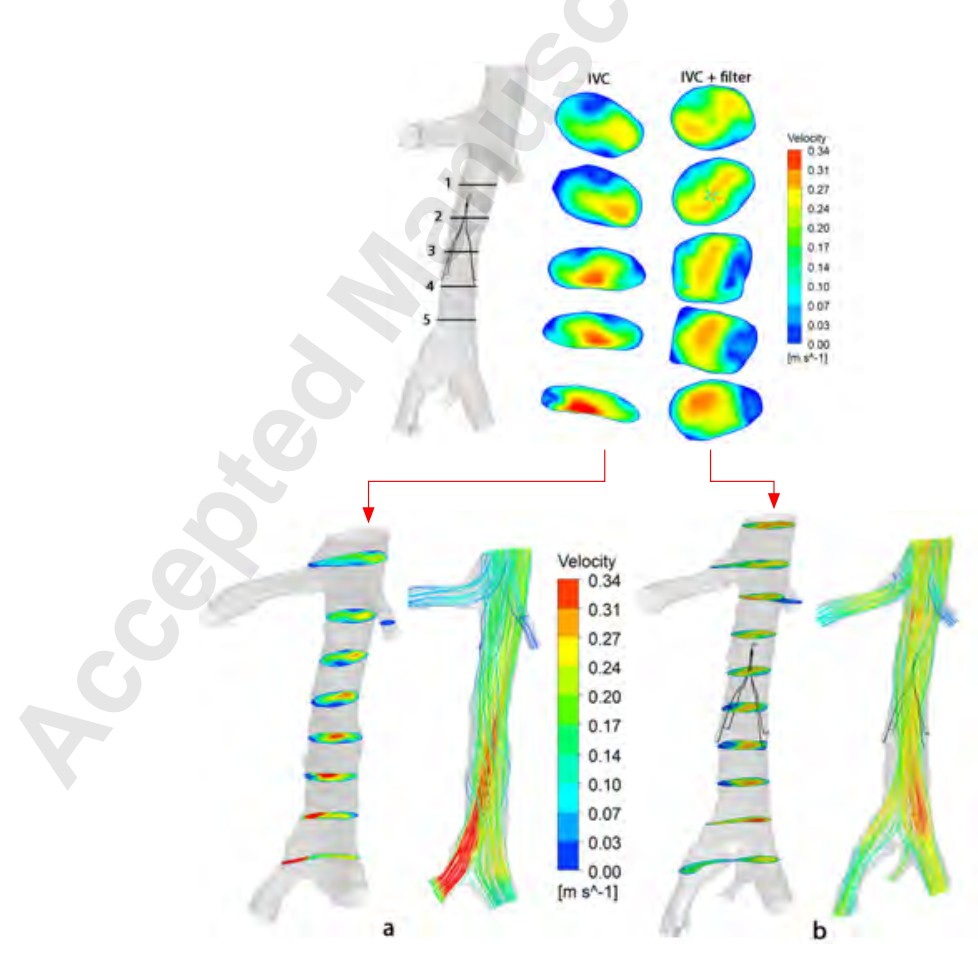

Fig. 6. Comparison between the vena cava fluid dynamics without filter (a) and after device deployment (b) during Valsalva. On the upper panel, velocity magnitude is displayed on transversal cut planes, on the lower panel three dimensional streamlines are shown. 
Copyright (ceccion reduces to $149.94 \mathrm{~mm}^{2}$, even for this condition, this is the wider opening surface. We start by presenting the results of the numerical simulation for neutral conditions. The vena cava is mainly affected by extravascular pressures coming from normal breathing, the intravascular pressure gradient being low. In particular, the flow dynamics of the vena cava is shown in presence as well as without a filter for this condition. In Figure 5 it can be seen from the progression of the various cross sections how the velocity field develops along the length of the vena cava. As stated in the literature, locations with low velocity, especially near the filter, may play an important role in clot capturing [4]. The results regarding the Valsalva manoeuvre are shown in Figure 6. The extreme decrease in the caval diameter during this manoeuvre (see Figure 2), causes an increase in the velocity magnitude compared to the normal breathing. The flow patterns are different for the considered cases. The maximum velocities experienced for both neutral conditions and Valsalva manoeuvre are $0.20 \mathrm{~m} / \mathrm{s}$ and $0.34 \mathrm{~m} / \mathrm{s}$ respectively. During Valsalva, the model with no filter shows a maximum velocity of $0.34 \mathrm{~m} / \mathrm{s}$ while the model that includes the filter has a peak velocity of $0.28 \mathrm{~m} / \mathrm{s}$. The highest value comes from the left iliac veins where the geometry presents an abrupt decrease in area as shown in Table 1. Furthermore, the dramatic increase in the velocity magnitude in the Valsalva model is a consequence of the considerable increase of the wall shear stress with respect to normal breathing as shown in Figures 7 and 8.

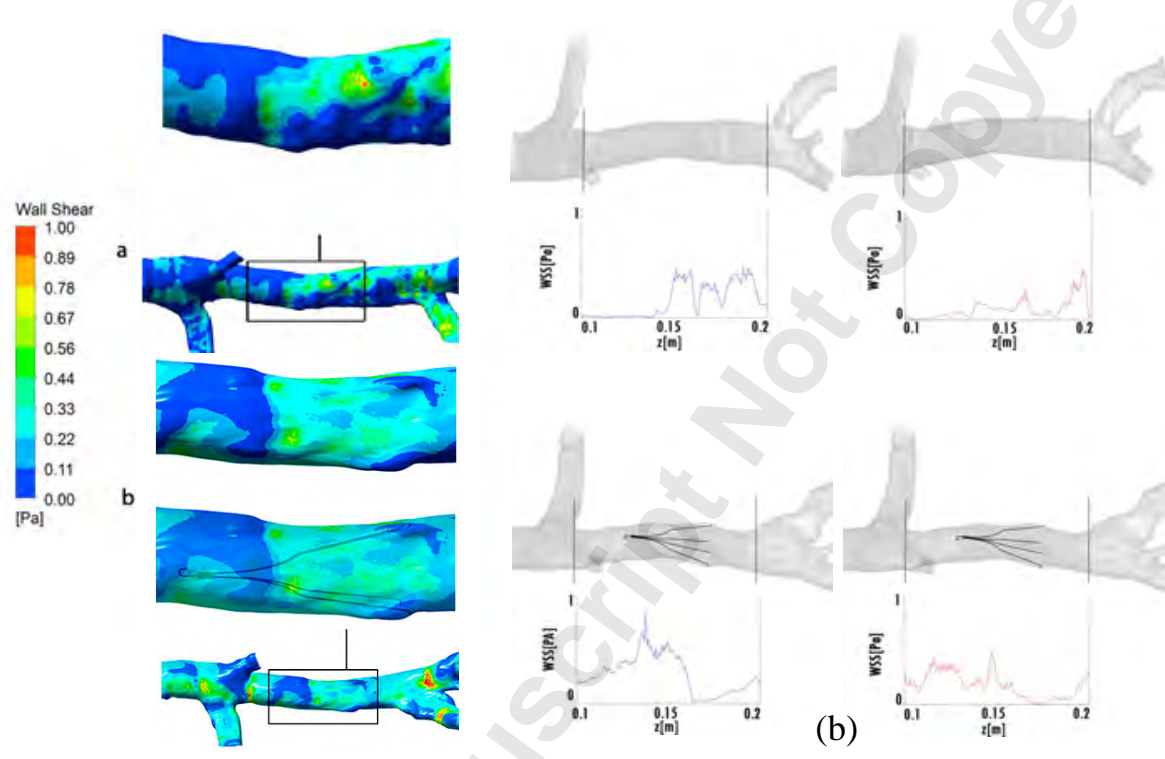

(a)

Fig. 7. Comparison between the vena cava WSS distribution without filter (left panel (a)) and after device deployment (right panel (b)) during normal breathing. The lines along which the WSS is displayed are obtained as intersection between the geometry and a longitudinal cutting-plane as sketched in the figure.

Comparing the WSS distribution with and without filter, it is visible that while during neutral conditions the presence of the filter is not affecting the WSS spatial distribution (see Figure 7), during Valsalva this distribution is quite altered (see Figure 8). In this case in fact, in the region near the filter, a drastic increase of the local WSS is shown (see details in the Figure 8). This increase, generated by the geometrical change of the vena cava as well as the associated increase in blood flow velocity may have a consequence for clot capturing as stated in literature [4]. The comparison between the flow patterns with and without filter (see Figure 5 and 6) seems to suggest that the presence of the filter for each single manoeuvre has little influence on the overall hemodynamics. Even if important changes are visible regarding the geometry of the vena cava (see Figure 2), the associated blood flow streamlines seems not to undergo any substantial change due to the presence of the filter. Under geometrical point of view, during Valsalva, the vena cava assumes an elliptical shape due to the extravascular pressure which makes the vena cava collapse. This sudden process is interrupted by the presence of the filter (see Figure 2). The IVCF, with its radial force, is capable of forcing a square vena cava section. Even the flow patterns are not massively affected by the filter, the consequence of this may be serious due to the penetration of the device struts through the caval walls. These locations are certainly subjected to higher stresses due to the extravascular pressure exerted on the filter legs. Also, due to the non uniform fluid dynamics at these locations, the spatial distribution of the WSS varies. For both cases, i.e. in presence and absence of the filter, we computed the WSS distribution along the vein walls as a function of the associated 

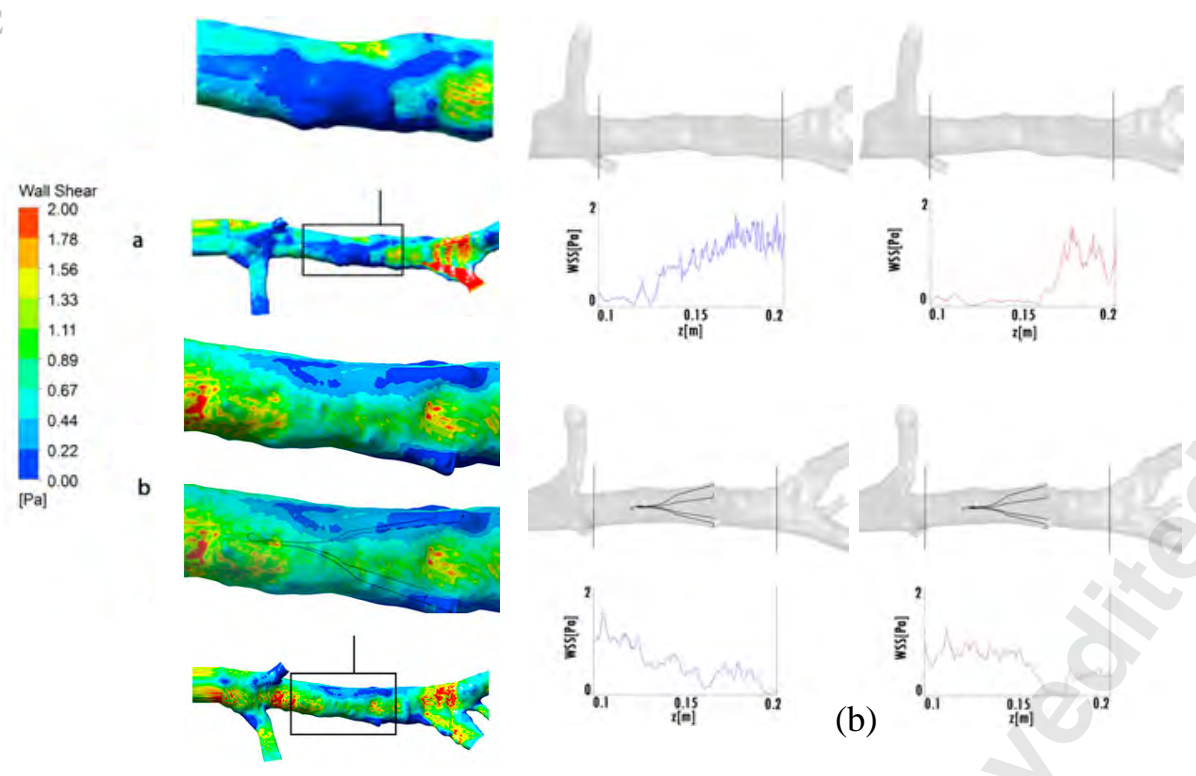

(a)

Fig. 8. Comparison between the vena cava WSS distribution without filter (left panel (a)) and after device deployment (right panel (b)) during Valsalva. The lines along which the WSS is displayed are obtained as intersection between the geometry and a longitudinal cutting-plane as sketched in the figure.

velocity field. Velocity changes induce local changes in the WSS which may play a role in tissue inflammation, growth and injuries, as largely demonstrated in literature, for example for cardiovascular stents [31-33]. The WSS distribution plotted on the vena cava wall (Figures 7 and 8 ) is also studied in the presence of the filter. In particular, the WSS is depicted along two lines obtained as intersection of a longitudinal cutting plane with the geometry (see detail on the Figures 7 and 8 ). The WSS variation is quite irregular in comparison with that sketched in other works [4]. This is basically due to the geometry of the vena cava which is patient specific in the present study while it is usually approximated as a perfect tube in studies in the literature $[4,12,14,15]$. This finding reinforces the importance of using patient specific geometries to accurate study the caval flow under different conditions. The WSS profiles suggest important differences due to the presence of the filter, during neutral as well as during Valsalva conditions. The local maximal WSS during normal breathing reduces when the filter is inserted, while during Valsalva an increase is shown. In both cases, the average wall shear stress increases. The WSS exactly at the strut site is 0 in all four cases (neutra with no filter, neutra with filter, Valsalva with no filter and Valsalva with filter) while at regions of the vena cava wall near the filter struts it shows an increased WSS value even though this may not have a strong influence on the wall itself due to the very small size of the filter leg. However, its importance may increase in the case of leg penetration into the wall, promoting the aforementioned fibrosis phenomena. Abnormal WSS distribution could also be related to non physiological tissue growth and may be associated to tissue inflammation [31,32]. Quantitatively, the maximum WSS for the neutral models is $1 P a$ while it is $2 P a$ for the Valsalva models. Finally, in the Table 4 we have computed the total drag force on the filter. In particular, the drag force is evaluated during natural breathing, considered as control condition for comparison purpose, and Valsalva. In the presented case (patient A), it is visible that the force acting on the filter during the control condition is quite small due to the stationary flow that characterizes the vena cava. During Valsalva an increase of this force is registered. The computed force is more than twice respect to that during natural breathing. The force acting on the device would help understand migration and could be useful (with more data/patients) on estimating load on the IVC filters during rest and Valsalva especially with increased clot capture.

Table 4. Computed drag force in $[N]$ during natural breathing (considered as control value for comparison purpose) and the Valsalva manoeuvre.

\begin{tabular}{|l|c|}
\hline & Drag Force $[N]$ \\
\hline \hline Patient A - control & 0.000880233 \\
\hline Patient A - Valsalva & 0.0020972 \\
\hline
\end{tabular}


The use of a vena cava filter for the treatment of thrombosis is still controversial and much discussed. Every filter type has its advantages and disadvantages. In this context, the presence of the filter during Valsalva may have serious consequences related to possible injuries to the vena cava wall. This computational flow study indicates firstly that renal vein flow has significant hemodynamic effects on blood flow during Valsalva irrespective of the presence or not of the IVCF. The renal venous flow in the Valsalva model dominates the overall flow patterns. The importance of the renal veins in the presence of IVCF was previously demonstrated in literature by the extensive work of Singer, Wang and coworkers $[11,12,14,15,17]$. The hemodynamics associated to the Valsalva manoeuvre is important taking into account two main aspects. First, the drastic geometrical variations of the cava section due to the extravascular pressure associated to this manoeuvre may cause alterations to the flow circulation, as experimentally found and debated in the literature [20,34].

The second important aspect is related to the damage that the filter may cause on the wall. Filter struts should be fixed at the vein wall in order to avoid migration phenomenon [35]. However, if the Valsalva is performed, the cava walls are forced against the filter legs which prevent the elliptical shape which characterizes Valsalva. There is therefore the risk of perforation of the wall. As confirmation, from the 3D reconstruction we have seen that the IVCF during Valsalva presents a square section at the filter location while this was almost elliptical in the absence of the device. When observing the sections described in Table 3 it can be noted that patient A performed a stronger Valsalva with respect to patient $\mathrm{B}$. This is not surprising since this manoeuvre is specific to each patient depending on the capacity of each single subject. The area reduction described in the Table 3 is in fact higher than that of patient $\mathrm{B}$. In particular, for patient $\mathrm{A}$ it is visible a reduction of around $70-75 \%$ while near the filter region (Area 4) the reduction is of 55\%. On the contrary, for the patient B (without filter) the reduction in terms of area was of about 50\% (average of $42-60 \%$ ) and in the filter region (area 4 ) the section reduction increased till 58\%. In conclusion, the presence of the filter drastically reduces the vena cava contraction during Valsalva near the region where the filter is usually placed. This might cause a relative increase of penetration of the filter legs in the caval walls due to the caval diameter reduction. In literature $[25,26,36]$ it has been reported that filter strut penetration has occurred in vena cava walls, suggesting that anomalous tissue growth may be promoted, including local tissue remodelling around the filter legs $[35,37,38]$. In addition, the retrievability of the medical device may be difficult in these circumstances. Using CT images we were able to build reliable models capable of providing important information on the cava geometries in different physiological conditions as well as showing the associated hemodynamics which increases the understanding of the blood flow circulation in the cava and related variables such as shear stress. These aspects reinforce the idea that numerical simulations applied to the vena cava, are not only useful for understanding the overall hemodynamics but could also be helpful for computing variables which can not be evaluated in vivo. The complex flow field generating during Valsalva and its implications are still debated in the literature [20,34]. Thus, the study of the associated hemodynamics may shed light on the physiology of this manoeuvre which it is necessary to perform in many clinical situations and which is often conducted by the patients before and after the insertion of an IVCF. In addition, the use of patient specific images to study vena cava flow allows to highlight changes in hemodynamics variables which cannot be obtained using simplified geometries.

Despite the fact that in recent years advances in IVC filters have proved to be successful in the treatment of thrombosis [38], and although new devices have been introduced, no currently available filter can be considered perfect $[7,8]$. The ideal filter should be easy to insert and remove, avoiding filter migration and able to anchor itself on the vena cava walls without complete penetration. For this reason, improvements in filter design to reduce the migration risk and to avoid wall damage are still required. Quantification of the stresses originated in the biological structure due to filter insertion may shed light on the mechanical aspects of tissue injury as well as the associated biological processes. In addition, quantification of shear stress in vein geometries could be performed to aid new filter designs and in order to improve clinical knowledge of medical devices and related outcomes. The fluid dynamics of the vena cava has been analysed using an attractive non invasive method providing an initial qualitative evaluation of the related hemodynamics which may be involved in the remodeling process of tissue growth as well as wall tissue damage, inflammation and fibrosis formation.

Clinical considerations which can be extracted from this study should be interpreted with caution, taking into account the limitations introduced in the numerical models. First of all, the presented numerical results should include an experimental/clinical validation that could improve the precision of the reported results. The clinical validation cannot be provided here due to the absence of data related to the analyzed patients and it is left for further studies and other analyses. Furthermore, additional patient data are necessary to extend this study. For example, the results might be influenced by the measured pressures. A larger number of measurements is therefore necessary in order to statistically observe intervariability of pressure between patients, especially when performing Valsalva. Moreover, the scans used for the reconstructions are quite rough ( $5 \mathrm{~mm}$ distance between consecutive slides). However, even though an increase in image quality would considerably improve the presented models, this would lead as well to health risk for the patients. Finally, a larger number of geometries should be reconstructed for performing a statistical analysis of the geometry variation during the considered manoeuvre for decreasing the uncertainties of the manual/automatic segmentation of the medical images. 
Accurate simulations of vena caval blood flow during different manoeuvres were carried out in order to understand the mechanisms involved in the return of blood to the heart. The hemodynamics of the vena cava during neutral and Valsalva conditions were analysed, observing the impact of a commercial antithrombotic filter on the associated geometrical and fluid-dynamics changes. The results of the proposed simulations show the different flows developing inside the vena cava during normal breathing and Valsalva manoeuvre as a direct consequence of the substantial geometrical variation of the cava geometry. The associated WSS developing inside the vena cava shows as a consequence important changes comparing these manoeuvres and the effect of the presence of the filter is assessed. In particular, although the filter considerably alters the geometry of the vena cava during both manoeuvres, especially during Valsalva, we found that its influence on the vena cava hemodynamics seems to be low. The importance of studying the caval flow by means of patient specific data is assessed evaluating variables such as the WSS. Increasing knowledge of the vena cava hemodynamics may help in the design of new filter prototypes which may address the failures of existing devices. Numerical models can be used to predict the mechanical consequences of clinical procedures and could be used in the future as a support decision tool, helping the preoperative planning of surgeries.

\section{References}

[1] Stein, P. D., Patel, K., Kalra, N., El Baage T, T., Savarapu, P., and Silberglet, A., 2002. "Deep venous thrombosis in a general hospital". Chest, 122, pp. 960-962.

[2] Anderson, F., Wheeler, H., and Goldberg, R., 1991. "A population based perspective of the hospital incidence and case fatality rates of deep vein thrombosis and pulmonary embolism: The worcester dvt study". Arch. Intern Med., 151, pp. 933-938.

[3] Hyers, T. M., Hull, R. D., and Morris, T. A., 2001. "Antithrombotic therapy for venous thromboembolic disease". Chest, 119, pp. 176S-193S.

[4] Swaminathan, T. N., Hu, H. H., and Patel, A. A., 2006. "Numerical analysis of the hemodynamics and embolus capture of a greenfield vena cava filter". Journal of Biomechanical Engineering, 128, pp. 360-370.

[5] Phillips, M. R., Widrich, W. C., and Johnson, W. C., 1980. "Perforation of the inferior vena cava by the kimraygreenfield filter". Surgery St. Louis, 87, pp. 233-235.

[6] Greenfield, L. J., Kyung, J. C., and Tauscher, J. R., 1990. "Limitations of percutaneous insertion of greenfield filters". Journal of Cardiovascular Surgery, 31, pp. 344-350.

[7] Grassi, C. J., Swan, T. L., and Cardella, J. F., 2001. "Quality improvement guidelines for percutaneous permanent inferior vena cava filter placement for the prevention of pulmonary embolism". J. Vasc. Interv Radiol., 12, pp. 137141.

[8] Athanasoulis, C. A., Halpern, J. A. K. E. F., Geller, A. C. W. S. C., and Fan, C., 2000. "Inferior vena caval filters: Review of a 26-year single-center clinical experience". Radiology, 216, pp. 54-66.

[9] Leask, R. L., Johnston, K. W., and Ohja, M., 2001. "In vitro hemodynamic evaluation of a simon nitinol vena cava filter: Possible explanation of ivc occlusion”. J. Vasc. Interv. Radiol., 12, pp. 613-618.

[10] Leask, R. L., Johnston, K. W., and Ohja, M., 2004. "Hemodynamic effects of clot entrapment in the trapease inferior vena cava filter". J. Vasc. Interv. Radiol., 15, pp. 485-490.

[11] Wang, S. L., and Singer, M. A., 2009. "Toward an optimal position for IVC filters: Computational modeling of the impact of renal vein inflow". Journal of Vascular and Interventional Radiology.

[12] Singer, M. A., Henshaw, W. D., and Wang, S. L., 2008. "Computational modeling of blood flow in the trapease inferior vena cava filter". Journal of Vascular and Interventional Radiology.

[13] Stewart, S. F. C., Robinson, R. A., Nelson, R. A., and Malinauskas, R. A., 2008. "Effects of thrombosed vena cava filters on blood flow: Flow visualization and numerical modeling". Annals of Biomedical Engineering, 36 (11), pp. 17641781.

[14] Singer, M. A., Wang, S. L., and Diachin, D. P., 2009. "Design optimization of vena cava filters: An application to dual filtration devices". Journal of Biomechanical Engineering, 132, pp. 101006-1.

[15] Singer, M. A., and Wang, S. L., 2011. "Modeling blood flow in a tilted inferior vena cava filter: does tilt adversely affect hemodynamics?". Journal of Vascular and Interventional Radiology, 22(6), pp. 229-235.

[16] Rahbar, E., Mori, D., and JE, J. E. M., 2011. "Three-dimensional analysis of flow disturbances caused by clots in inferior vena cava filters". Journal of Vascular and Interventional Radiology, 22(6), pp. 835-842.

[17] Ren, Z., Wang, S. L., and Singer, M. A., 2012. "Modeling hemodynamics in an unoccluded and partially occluded inferior vena cava under rest and exercise conditions". Medical \& Biological Engineering \& Computing, 50(3), pp. 277287.

[18] Aycock, K. I., Campbell, R. L., Manning, K. B., Sastry, S. P., Shontz, S. M., Lynch, F. C., and Craven, B. A., 2014. "A computational method for predicting inferior vena cava filter performance on a patient-specific basis". Journal of Biomechanical Engineering-Transactions Of The Asme, 136(8), p. 081003. 
Copyright (19] G Greenfield, L. J., and Proctor, M. C., 1992. "Experimental embolic capture by asymmetric greenfield filters". J. Vasc. Surg., 16, pp. 436-444.

[20] Kuzo, R. S., Pooley, R. A., Crook, J. E., Heckman, M. G., and Gerber, T. C., 2007. "Measurement of caval blood flow with mri during respiratory maneuvers: Implications for vascular contrast opacification on pulmonary ct angiographic studies". American Journal of Roentgenology - Chest Imaging, 188, pp. 839-842.

[21] Kowallick, J. T., Joseph, A. A., Unterberg-Buchwald, C., Fasshauer, M., Van Wijk, K., Merboldt, K. D., Voit, D., Frahm, J., Lotz, J., and Sohns, J. M., 2014. "Measurement of caval blood flow with mri during respiratory maneuvers: Implications for vascular contrast opacification on pulmonary ct angiographic studie". British Journal of Radiology, 1042(87), p. 20140401.

[22] Taylor, D., 1996. “The valsalva manoeuvre a critical review”. SPUMS Journal, 26, pp. 8-13.

[23] Nishimura, R. A., and Tajik, A. J., 1986. "The valsalva manoeuvre and response revisited". Mayo Clin Proc, 61, pp. 211-217.

[24] Nicolás, M., Palero, V. R., Peña, E., Arroyo, M. P., Martínez, M. A., and Malvè, M., 2015. "Numerical and experimental study of the fluid flow through a medical device". International Communications in Heat and Mass Transfer, 61, pp. 170-178.

[25] Laborda, A., Kuo, W. T., Ioakeim, I., De Blas, I., Malvè, M., Lahuerta, C., and De Gregorio, M. A., 2015. “Respiratoryinduced haemodynamic changes: A contributing factor to ivc filter penetration". Cardiovascular Interventional Radiology, 38(5), pp. 1192-1197.

[26] Laborda, A., Malvè, M., De Blas, I., Ioakeim, I., Kuo, W. T., and De Gregorio, M. A., 2014. "Influence of breathing movements and valsalva maneuver on vena caval dynamics". World Journal of Radiology, 6(10), pp. 833-839.

[27] Sastry, S. P., Kim, J., Shontz, S. M., Craven, B. A., Lynch, F. C., Manning, K. B., and Panitanarak, T., 2013. ImageBased Geometric Modeling and Mesh Generation, Vol. 3, pp. 223-249, Springer, ISBN 978-94-007-4255-0.

[28] Cheng, C. P., Herfkens, R. J., and Taylor, C. A., 2003. "Inferior vena cava hemodynamics quantified in vivo at rest and during cycling exercise using magnetic resonance imaging”. Am J Physiol Heart Circ, 284, pp. 1161-1167.

[29] Ansys Inc., 2012. "Ansys CFX Solver Theory Guide". Release 14.5.

[30] Murray, C. D., 1926. "The physiological principle of minimum work, the vascular system and the cost of blood volume". Proceedings of the National Academy of Sciences USA, 12, pp. 207-214.

[31] Moore, J. E., and Berry, J. L., 2002. "Fluid and solid mechanical implications of vascular stenting". Annals Biomedical Engineering, 30, pp. 498-508.

[32] Malek, A. M., Alper, S. L., and Izumo, S., 1999. "Hemodynamic shear stress and its role in atherosclerosis". J. Am. Med. Ass., 282, pp. 2035-2042.

[33] Balossino, R., Gervaso, F., Migliavacca, F., and Dubini, G., 2008. "Effects of different stent designs on local hemodynamics in stented arteries". Journal of Biomechanics, 41(5), pp. 1053-1061.

[34] Wexler, L., Bergel, D. H., Gabe, I. T., Makin, G. S., and Mills, C. J., 968. "Velocity of blood flow in normal human venae cavae". Circulation Research, 23, pp. 349-359.

[35] García, A., Lerga, S., Peña, E., Malvè, M., Laborda, A., De Gregorio, M. A., and Martínez, M. A., 2012. "Evaluation of migration forces of a retrievable filter: Experimental setup and finite element study". Medical Engineering \& Physics, 34, pp. 1167-1176.

[36] Laborda, A., Lostalé, F., Rodríguez, J. B., Bielsa, M. A., Martínez, M. A., Serrano, C., Fernández, R., and De Gregorio, M. A., 2011. "Laparoscopic demonstration of vena cava wall penetration by inferior vena cava filters in an ovine model". Journal of Vascular Intervention Radiology, 22, pp. 851-856.

[37] De Gregorio, M. A., Gamboa, P., Gimeno, M. J., Madariaga, B., Tobío, R., Herrera, M., Medrano, J., Mainar, A., and Alfonso, R., 2003. "The Günther Tulip retrievable filter: prolonged temporary filtration by repose- tinning within the inferior vena cava". Journal of Vascular Intervention Radiology, 14, pp. 1259-1265.

[38] De Gregorio, M. A., Laborda, A., Higuera, M. T., Lostale, F., Gómez-Arrue, J., Serrano, C., Martínez, M. A., and Viloria, A., 2008. "Removal of retrievable inferior vena cava filters 90 days after implantation in an ovine model: is there a time limit for removal?". Arch Bronconeumol, 44, pp. 591-596.

\section{Achnowledgements}

This study was supported by the CIBER-BBN financed by the Instituto de Salud Carlos III, by the Spanish Ministry of Science and Technology through the Research Projects DPI-2010-20746-C03-01 and DPI-2013-44391-P and by the Spanish Ministry of Economy and Competitiveness through the Research Project DPI-2016-76630-C2-1-R. The experimental study was supported by the Instituto de Salud Carlos III, through the research project PI08/1424 and was performed by the Minimally Invasive Techniques Research Group (GITMI) of Aragón Government. 